\title{
TELEMEDICINE: THE FUTURE OF MEDICINE A CASE STUDY OF TELEMEDICINE APPLICATIONS WITHIN THE UNITED STATES
}

\author{
Suzanne Spradley \\ National Clinical Systems Development \\ Kaiser Permanente \\ United States of America
}

\begin{abstract}
Telemedicine is the interactive audio-visual communication between health care providers and their patients. The paper describes various aspects of telemedicine in the US. Telemedicine system can utilise video conferencing, voice mentoring, mobile high bandwidth communications systems and digital imaging. They claim to improve the quality of emergency care, provide access to specialty care regardless of location and provide better overall medical decision support. Examples are cited to show that telemedicine is used for emergency consultation, mentoring at a distance, providing access to medical expertise in remote rural and expensive-to-serve areas, and for patient and medical education. It is claimed that potential savings can include provider time and travel, patient time and travel, savings from reductions or substitutions in personnel needed, the reduction of redundant tests, and treatment early in the course of the disease when treatment is less costly.
\end{abstract}

\section{TELEMEDICINE: INTRODUCTION}

Telemedicine is the interactive audio-visual communication between health care providers and their patients. It is an application, not a technology. Telemedicine provides global, real time access to quality care though integrated communications and information technologies. A telemedicine system can utilise video conferencing, voice mentoring, mobile high bandwidth communications systems and digital imaging to improve the quality of emergency care, provide access to specialty care regardless of location and provide better overall medical decision support. The simplest definition of telemedicine is that it uses multimedia (voice, video and data) technology to deliver medical services and began with the simplest technology: the telephone. As the cost of bandwidth decreases and improvements in video and data compression standards increase, the number and types of medical services that can be delivered from a distance will expand to include virtually every specialty. It is the extension of medical information, with all its graphic intensity, from its source, the hospital, to health care professionals in surrounding areas and ultimately to the home. Since telemedicine was first used in 1959, when $\mathrm{x}$-ray images were transmitted across telephone lines, technology has advanced making telemedicine more affordable and clinically useful. Telemedicine can also be defined as a link between under-served areas and a major referral centre 
such as the Mayo Clinic and Yale University in the East. Nationally, a disproportionate number of deaths occur in rural areas due to unintentional injury, motor vehicle accidents and other trauma-related injuries. The state of Colorado suffered 97.7 such deaths per 100,000 population in rural and frontier counties in 1990, compared to 60.4 per 100,000 population in urban areas (Caldwell, 1995).

\section{TELEMEDICINE: WHAT TECHNOLOGIES ARE BEING USED? HOW DOES IT WORK?}

Current telemedicine technology allows for a spectrum of capabilities. Still pictures over a phone line, augmented by direct verbal communication, is presently user-friendly, economical and has a proven utility in the prehospital care arena. The compressed audio-visual technology allows direct and instantaneous visual and audible interactions with the patient. Auscultation, visualising tympani membranes and fundoscopic exams can be performed accurately and in real time.

Telemedicine uses a hybrid technology incorporating elements of television, telecommunications, computers and engineering. Services can be delivered on a combination of technologies with a variety of equipment. Most telemedicine applications require interactive voice and video, the quality of which varies according to the delivery system. At the lowest end, several companies make a slightly more sophisticated version of the telephone which allows audio and still images to be sent between sites via conventional telephone lines through the use of a handset, a camcorder or a built-in camera at one end and a monitor at the other end. The Internet also provides a platform for sending data, text, still images and limited full motion video. H.320 standards provide for simultaneous audio (G.700), video (H.261) and data transfer (T.120) using a minimum of $56 \mathrm{Kbps}$ line. Compatibility with H.320 ensures interoperability with the widest range of third-party teleconferencing systems. MPEG1 supports compression of VHS quality video and $\mathrm{CD}$ quality audio into a $1.5 \mathrm{Mbps}$ bitstream or higher quality (ultrasounds). MPEG2 supports compression of wide-ranging quality video and audio beyond that of HDTV into a bitstream up to $100 \mathrm{Mbps}$.

At the higher end of the technology scale are dual monitor videoconferencing systems (one monitor displays the sending image and one the receiving image) which are equipped with microphones, built in cameras, a video codec which compresses the video into digital form and a user interface. Graphical user interfaces are implemented with toolbars to quickly access common image processing functions such as zoom, shrink, horizontal flip, etc. As an image set is opened at either site, it is immediately transferred to the remote site and the two images are synchronised. Two separate cursors are controlled by the local and remote user respectively. Multiple image sets can be open at once and real-time video can be transferred simultaneously with image manipulation (Cabral et al 1995). Digital scanners, microscopes, VCRs, telestrators (annotators) and 
electronic medical instruments such as stethoscopes and otoscopes can be added to these systems as required. The speed of transmission largely determines the quality of the picture. On the telephone lines, these systems can run from $112 \mathrm{Kbps}$ to $1.544 \mathrm{Mbs}$, all at 30 frames/second. A codec compresses the video signal at one end and decompresses it at the other. Video-conferencing at speeds of $1 / 2 \mathrm{~T} 1$ or higher give full motion, real time, near-broadcast quality video. Full broadcast quality (uncompressed video) can only be achieved using a satellite.

Developing a reliable delivery system has been slow, which contributed to the cautious pace of telemedicine in the early 1990s. Reliability is still an issue for some aspects of some technologies. Redundancy of equipment and/or personnel protects data and ensures patient safety. Although the technology appears as simple and familiar as turning on a TV set, in fact multiple technical elements are involved and users must be trained to operate the equipment.

East Carolina University recently opened the first Telemedicine suite in the country, comprising four rooms specifically designed for telemedicine purposes, which include video conferencing capabilities and specially developed diagnostic tools such as a digital stethoscope, a dermoscope and a graphics camera for viewing X-rays. These rooms are connected to an integrated network that include remote hospitals, nursing homes, prisons and Health House. Health House is a 3000 sq. foot house with $100 \mathrm{Mb} / \mathrm{sec}$ Ethernet designed into the structure, which accommodates health diagnostic, monitoring and therapeutic applications. Two-way full motion telemedicine applications are designed to facilitate health compliance and support independence at home, provide 24-hour access to assistance, allow for follow-up on health issues, detect early problems, provide triage to appropriate care, and avoid unnecessary utilisation of health facilities.

\section{TELEMEDICINE: WHERE IS IT USED?}

Telemedicine breaks down geographic barriers and will save a lot of money very quickly by treating many patients on site rather than in an expensive hospital setting; it will improve patient care by giving health care providers access to teaching medicine resources, and it will target services to populations which have been hard to reach (remote rural areas), expensive to serve (prisons, mental institutions), and historically neglected (urban poor).

\subsection{Emergency consultation}

Industry has begun to use this communication technology on oil rigs and cargo ships. Injured workers can have treatment begun while waiting for transportation to a hospital. This decreases the delay in their acute medical treatment. Telemedicine has been used in Croatia and Somalia to help treat 
the injured and the sick. These countries have invested a reported \$US 70 million in developing this technology and private industries such as AT\&T and V-TEL are reported to be investing up to \$US 1 billion in developing advanced communication technology. There are 13 federal agencies and 40 state agencies that are involved in setting up the infrastructure to make telemedicine accessible, cost effective, user-friendly and clinically useful. Telemedicine has been used to provide medical consultation during natural disasters such as earthquakes. University hospitals are providing second opinions and continuing medical education to community hospitals as far away as the Middle East. Patients may be examined by a local doctor or nurse, while a physician thousands of miles away participates via two-way television. Patients could join both practitioners in discussing the medical condition and methods of treatments. To date, satellite-based speech pathology consultation, satellite-based monitoring of patients receiving dialysis, satellite transmission of EKGs, radiographic images is becoming common.

\section{2 $\quad$ Telementoring}

A large University hospital links to hospitals around the country, allowing their surgeons to guide or mentor a less experienced surgeon at a distant site during a laparoscopic surgical procedure. Fewer than $10 \%$ of US surgeons perform advanced laparoscopic surgery and there is an estimated $43 \%$ failure rate on initial laparoscopic procedures. Telementoring has sharply reduced or eliminated failures (Holaday and Swett 1995).

\subsection{Remote rural and expensive-to-serve areas}

In the future, telemedicine may provide rural hospitals with the subspecialty expertise of a university hospital, allowing the patient to receive treatment in their local hospital. Patient vital signs can be transmitted to a database and analysed with data from nearly 5 million medical records. The patient could then receive a personalised report of his or her risk for certain diseases such as diabetes, heart attack, etc. If a patient has these conditions, followup care and monitoring are important (McGlinch 1996). A patient's EKG or glucose level could be recorded and transmitted directly to the hospital from the patient's home. These decrease the patient's cost of travelling and also decrease the amount of time and anxiety waiting for the sub-specialist's input. It allows rural hospitals to increase their census since they will maintain the ability to care for all degrees of sick persons, and nursing homes will be able to treat patients without sending them to an acute care hospital. Prisoners, who are both costly and dangerous to transport, can receive their treatment at the prison with subspecialty consultations via telemedicine. Third world countries and poor rural areas could potentially have subspecialty advice upon request. 


\subsection{Patient education}

Nurses, dieticians, physical and occupational therapists use telemedicine to deliver cost-effective, personalised information and follow-up patient care.

\subsection{Medical education}

Professional isolation is a major reason why rural communities have difficulty recruiting and retaining physicians. Telemedicine helps keep doctors up to date with the latest findings in their field and remain in contact with colleagues. By providing such linkages, telemedicine can help resolve the uneven distribution of physicians in our country.

\section{TELEMEDICINE: COST SAVINGS}

Potential savings can include provider time and travel, patient time and travel, savings from reductions or substitutions in personnel needed, the reduction of redundant tests, and treatment early in the course of the disease when treatment is less costly.

Baylor College of Medicine: A representative for the urban telemedicine project reported that if the base system cost were $\$ U S 50,000$ per site, cost justification could be reached at 100 hours of use per year (Global Telemedicine Report 1994).

Medical College of Georgia: This program found that $81 \%$ of patients seen over telemedicine did not require transfer to secondary or tertiary carecentres. In Georgia, the cost differential between rural hospital beds and MCG beds is \$US 800. In addition to that saving, telemedicine allows savings in transportation, increased productivity, and decreased hospitalisation days from treating a patient at an earlier stage. MCG reported that if rural hospitals were to retain telemedicine patients, the increase of a single patient per day to the rural hospital census would represent a net cash flow of \$US 150,000 per year for the hospital (Moore 1996).

In 1992 Arthur D. Little, Inc. Consultants reported that the Texas Telemedicine Project in Austin, Texas, produced "at least a 14\% savings" over more standard medical services. According to their model, reduction in overhead and travel time was the primary sources of savings from teleconferencing (said to approximate \$US 131.6 million per year nationally). Over two years a total 2,696 patients were treated. Six livers were reported saved. Equipment payback time was estimated at 3.5 years. In addition, Lee Memorial Hospital reported \$US 8,000 profit per quarter. Increased revenues of $\$$ US 37,000 were attributed to reinstituting infant deliveries and retaining local patients. Austin Diagnostic Clinic reported a net profit of \$US 9,000 per quarter; a cost/benefit ratio of 2.25 ; and equipment payback in 2.2 years. 
There are additional cost savings in professional and continuing education carried on by teleconferencing. Administrative cost savings include using telemedicine for administrative meetings, interviews of candidates for hiring, quality control meetings and training, obviating travel costs and lost productivity. Revenue has been generated from re-selling bandwidth and from selling video-conferencing meeting services to others. HBO \& Company provides health care information systems and services to 3,500 hospitals internationally. Over a 7-month period in 1993, HBOC reported \$US 290,927 saved in travel, excluding the productivity savings avoiding 3,888 employee travel hours.

\section{TELEMEDICINE: BENEFITS (PERCEIVED AND REALISED)}

Many of the benefits derived from health care systems cannot be measured in purely monetary terms. Factors such as equity of access, quality of care and the clinical support environment have an enormous indirect effect on the outcomes for both patients and clinicians. For patients in the TARDIS Telemedicine project, as a specific example, and in telemedicine project in general, the project had the potential to provide:

- improvement in outcomes due to assistance provided by the tertiary hospital;

- $\quad$ reduced need for transfer to a metropolitan hospital (of the 700 admissions per year to the ICU, $10 \%$ are transfers from other hospital(s), eliminating the significant disruption for families that occurs when they accompany transferred patients to metropolitan hospitals.

The ultimate goal is a revision of the health paradigm from "the patient going to the health service" to the "health service going to the patient" (TARDIS 1998).

\section{OUTCOMES (TARDIS EXAMPLE)}

- An Operational ICU telemedicine service for clinical and educational use.

- Modular telemedicine solutions in an integrated environment.

- A methodology for evaluating telemedicine requirements and translating them into a system specification. Note: For any telemedicine application to be successful, it must be based upon an understanding of the clinical environment into which it will be introduced. The first stage of the TARDIS project was to understand the communications and interactions that currently occur within ICUs. The overall intention was to develop a model of how people best work 
cooperatively and reflect that within the telemedicine solution. This process of developing the model forms the basis of a more universal methodology for evaluating telemedicine requirements.

- Blueprint for new clinical methodologies. After implementation of the ICU telemedicine system, and after developing the evaluation methodology, TARDIS was able to learn and create blueprints of the way clinicians collect, interpret and communicate knowledge. From this we may be better positioned to understand the role of technology in this process and how it may be applied for the benefit of health care delivery (TARDIS 1998).

\section{TELEMEDICINE: CURRENT PROJECTS IN PLACE}

Remember that the goal of telemedicine is that the physician or health care provider would be able to interact with the patient much like a face-to-face clinic visit or for the physician to provide mentoring or subspecialty services to junior or general medicine physicians. At East Carolina University, the telemedicine network is a scaleable hybrid of T1, ATM, POTS, Microwave and SW56 transmission modes. A variety of compression engines have been tested, deployed and integrated in to a multi-channel bridge that interconnects all of these technologies and allows cross-platform communication.

A primary component of telemedicine is an optimised physician workspace in which specialty physicians conduct consultations with patients at spoke sites. The physician sits in a custom-designed 6 by 12 foot sound-proofed booth and has a range of tele-diagnostic tools available.

An application, which connects small, rural hospitals to a tertiary care centre, must include tele-radiology services so that the tertiary care centre can receive high-resolution images of a patient's radiological studies. This application would use high-speed video, audio and data communications over ATM/SONET. At ECU, the network connects all four medical schools in North Carolina and has been designed to protect patient privacy using dedicated $27 \mathrm{Mbps}$ circuits for each telemedicine link. The links provide full support for all telemedicine tools and include far-end camera controls and full-motion, 30-frames-per-second video.

A new component at ECU, a still-image transfer system that uses standard telephone lines, illustrates the scalability of telemedicine. Compared with the application described above which uses high bandwidth and the most expensive technology available, this application uses standard telephone service and equipment with a total cost of less that \$US 10,000. Using a video telephone, a primary care physician can transmit dermatological or orthopaedic images to specialty physicians. The videophone includes an 
image storage system, so multiple patient cases can be transmitted in a store-and-forward manner (Balch and Tichenor 1997).

\subsection{Denver health and hospitals}

Denver Health and Hospitals has targeted the export of telemedical care by board-certified emergency physicians, trauma surgeons, radiologists, neurosurgeons and other specialists to rural Colorado. The expert intervention by these physicians in the hour immediately after injury, such as automobile accidents, has routinely saved lives and prevented permanent disability. With the technology available, these vital services can be extended to patients injured in remote parts of the state and region, dissolving traditional geographical barriers. Colorado's mortality rates from unintentional injury are some of the highest in the US, largely as a result of the state's rugged terrain and extensive skiing, tourism and recreation industries (Hinsdale, no date).

The Telemedical Trauma System is designed to include:

- integration into existing hospital information systems;

- $\quad$ integrated display of patient monitoring and imaging devices;

- wireless transmission for personal digital assistants;

- the development of interoperability computerised patient records;

- any-to-any desktop video-conferencing and other applications sharing.

\subsection{Prison Population}

Inmate \#35271 has an unusual skin rash that the prison doctor cannot diagnose. The prison administration does not want to be sued for inadequate medical care for its prisoners, but it does not want to run the security risk of transporting a dangerous felon to an outside specialist. Bringing the specialist to the prison would also present problems. The solution is a telemedicine link with a University school of medicine (Blanton 1995). In a special telemedicine booth a hundred miles away, a dermatologist sits in front of a video console and interviews the patient while directing the prison nurse where to point the tiny dermatology camera. The condition is diagnosed and a course of treatment is prescribed. "It costs the State of North Carolina \$US 700/day to transport an inmate outside the prison for medical care. But via telemedicine, prisoners at the state's largest penitentiary can be seen by medical specialists at East Carolina University for \$US 75/consult." (Lenn 1994)

An important issue in developing telemedicine has been in states such as California where providing prison health care can cost over \$US 380 million/year. With the implementation of such laws as 'Three-strikes you're out', prison populations will increase as will the cost of prison health care. In 1990, a prison inmate sued the state of Florida over inadequate health 
care and won a \$US 1 million settlement. That prompted Florida to begin prison telemedicine on a pilot basis. The next year, North Carolina's largest prison contracted with East Carolina University to provide telemedicine services. Since that time, physicians from the medical school have provided more than 350 consultations with the prison.

\section{DISCUSSION}

Over the next 10 years, we will see health care become less doctor-centred and more community- and family-centred. Medicine itself will become less of an art and more fact-based. Yet, at the same time it will come to feel more humane; less about the hardware and the powerful machines watched over by highly trained personnel, and more about shared information. The shift away from traumatic, intensive, expensive hospital-centred care is already occurring as hospitals close with emphasis shifting toward early-aspossible, preventive, long-term, intimate, inexpensive application of information in the community and the family.

Per capita, Americans have the most expensive health care system in the history of the world, yet we are less satisfied with our health care than cities in any other developed nation. And we are not the people with the best longevity. Our infant mortality is $22 \mathrm{nd}$ in the world, maternal mortality 26th. The information which is handled in health care today is face-to-face, filled-out insurance forms, notes scribbled on medical charts. In fact, much of the information is never recorded at all. And, even if it is recorded, health information experts estimate that $25 \%$ of this information does not get where it is needed (it is lost, mis-routed, or not sent in time) and must be regenerated. Health-oriented telecommunications can fix such information problems while making much larger changes possible.

For decades, medical technology has focused on ways to bring more and better information to the doctor. Now it is finding ways to release the doctor, to make more efficient use of the physician's time so that specialists can fan out to where their expertise is needed: battle zones, prisons, inner cities, rural areas and mental hospitals. There is no waiting to speak of, no overnight messengers needed to transport x-rays, CAT Scans and MRIs, and less travel is required between hospitals and offices for doctors and their patients.

The biggest hurdle to broader use of telemedicine is its high expense. Equipment, including computer-controlled multi-link switching devices, bandwidth, controllers, electronic bridges and 32" high-resolution TV monitors, costs about \$US 50,000 per site says North Shore's Dennis Skahill, who has set up nearly 200 telemedicine conferences that have been conducted over North Shore's Distance Learning Network. Telemedicine requirements are more extensive than standard video-conferencing set-ups that are used to tie together many corporate offices. It is o.k. for sales people 
to see a mildly fuzzy image of the company president, but medical graphics, $\mathrm{x}$-rays, CTs and MRI scans must be much clearer to be of value.

There are currently telementoring helmets that can be worn by battle medics or civilian paramedics. An EMT puts the helmet on, with its imbedded video cameras and microphones, and the doctor back at the hospital can see and hear what the EMT sees and hears, coach the EMT, help the EMT to do far more complex things in those first critical moments to try and stabilise the patient. This same technology, in the future, will be able to put a doctor at every bedside in every rest home, in every ambulance and even in our own homes.

Most medical practice/protocol has not been rigorously tested or examined to determine when the procedures are needed, when they work, and what would make them work better. To do that, you need what has only recently become available: massive numbers of computers across the country, reporting huge amounts of data from actual cases. It is pushing hospitals and doctors toward fully computerised patient records and clinical systems. Most clinical information - vital signs, doctors' observations, nurses' notes - is still recorded on paper. And information from a stay in one hospital does not usually link up with a stay in a different hospital. And the hospital records do not link up with the record from your doctor's office, clinic or outpatient surgery centre. Arthur D. Little consulting conducted one study that stated that the US could save 15 billions dollars every year by digitising everyone' medical records and putting them on-line, available to anyone with the right pass codes anywhere in the country. On the research end, aggregating vast amounts of clinical information on-line will lead to queriable research databases able to provide physicians and patients information about outcomes treatments, drug research and decision-making skills. For example, the standard dose of drug $\mathrm{X}$ is $10 \mathrm{cc}$, but the experience in the database shows that with an elevated blood count in a male over age 60 with lung complications, $5 \mathrm{cc}$. would be better.

In health care, costs can be cut and a better job can be done if people are taught how to improve their health, if disease is detected as early as possible, and if people are cared for at the lowest appropriate level of intensity. Keep colds and back aches out of the Emergency rooms; keep the Intensive Care free of patients with ailments that a little prevention could have caught. Telecommunications has powerful potential to help with patient education in the home.

Two-way switched broadband fibre-optic networks that extend into people's home would allow a lot more of the frail elderly and the chronically ill to live independently, rather than in convalescent homes. Less-trained personnel could visit them at home and link directly into the technological support and expertise of the hospital, hooking up to share vital signs, advice and a direct look at the patient with the patient's doctor. 
Most people (estimate: $50-80 \%$ ) entering the health care system don't really need a doctor's care. Most health problems (estimate: 70-80\%) can be cared for by the patient themselves if provided with the right information. Most people (estimate: 60\%) who seriously need a doctor's care, enter the system too late, ending up needing care that is far more intensive, painful, expensive and dubious than they would have needed earlier. Almost all firstline health decision (estimate: 95\%) are made outside the health care system, by the individual with the help of family and friends. Most of a good diagnosis (estimate: 70\%) depends on what the patient tells the doctor. If people were given an easy way to obtain accurate information about their own health at home, large amounts of money could be saved. A doctor at a rural hospital who uses telemedicine to consult a specialist in the city saves the patient (and the insurance company) the cost of travelling to the city and checking into a hospital for a separate consultation. If one patient per day checks into the rural hospital instead of travelling to the city, the rural hospital will be able to pay for the telemedicine equipment within a couple of years. Because of these savings, many governmental and private companies will be interested in providing health-oriented telecommunications for free. The Harvard Community Health Plan, for example, reduced the number of people visiting its clinics by $5 \%$ by using a computer-based triage and education system. When people come to the clinic, they sit down at the computer screen, answer a few simple questions and the system then tells the patients whether they really need to see a doctor.

Currently, expectations of telemedicine are unreasonably high. The smooth integration of multiple real-time media streams is still a technical challenge and the required tele-communications infrastructure is still maturing, especially in rural areas.

The legal implications of using telemedicine have not yet been fully explored and resolved. Physicians practising in multiple states are required to become licensed in each state. Patient confidentiality needs to be maintained across public networks. And the question of malpractice liability in the case of misdiagnosis and misconsultation using telemedicine is difficult (Kim et al 1995).

Successful deployment of telemedicine for home health care assumes that the technology will be accepted and that is will be used appropriately. Social researchers are questioning both assumptions while advocating increased patient control over the equipment sited in their homes. Industry analysts predict that $20 \%$ of all home health care visits will be delivered via telemedicine by 2001 , fuelled by cost savings as compared with home nursing visits, managed-care payment schemes and decreasing telecommunications costs. Published surveys of patients and home health care providers tend to focus on satisfaction or convenience, but none have questioned whether it is even appropriate to adapt hospital-based medical care processes to home-based environments. Another social issue to be considered is privacy. Issues of patient privacy go beyond consent forms, and relate directly to patient control of the equipment sited in their homes. "The right to control information about oneself is essential to growth," said 
Malcolm Fisk, a social policy researcher at John Moores University in Liverpool, U.K. "That means that the users should have the right to change parameters or switch off or disconnect equipment and that cameras are not to be used except in clearly defined circumstances that have been fully agreed upon by the patients." (Dakins 1997)

Telemedicine may also extend a hospital's market as these technologies expand the range of specialties available in rural communities and thus potentially improve the quality of rural health care and it also potentially increases the number of services a hospital is able to offer. It may also expand the geographical area in which a hospital offers its services, enhancing its competitiveness. Telemedicine does not change how physicians at the facilities practice medicine, but it makes hospitals competitive because patients demand state-of-the-art technologies (Emery 1997, Jones 1997).

The success of telemedicine ultimately revolves around physician acceptance and being true to the way physicians practice medicine. The early prison experience provided the opportunity to work out the ergonomics and human factors to make telemedicine work. Telemedicine must be designed to be easy for the physician to use. It is intended to assist clinical practitioners in getting their work completed. Consultations do not last longer over the telemedicine network and both physicians and patients involved in a rigorous evaluation are satisfied by outcomes. As individuals increasingly interact with health care professionals from their living rooms, telemedicine offers health insurers, particularly those serving rural areas, a tool for improving access to care, reducing costs, and making care more convenient. As Marvin P. Mitchell, Administrator of the Mayo Telehealthcare Center in Rochester says, linking geographically separated physicians and patients means that, "physicians would spend their time staring through their windshields for two or three hours. (Now) They will be seeing patients instead." (Flower 1993)

\section{REFERENCES}

Appleby, C. (1995) A Prison Plugs In. Hospitals \& Health Networks January 1995.

Balch, D. C., Tichenor, J. M. (1997) Telemedicine expanding the scope of health care information. Journal of the American Medical Informatics Association 4(1) Jan/Feb 1997.

Blanton, D. (1995) Telemedicine. The Futurist 29, September 1995.

Cabral, J.E., Parsons, D.M., Lipski, G.L., Kirchdoerfer, R.H. (1995) SeaHawk: a telemedicine project in the Pacific Northwest. http://icsl.ee.washington.edu/projects/gsp9/spie95/seahawk/ August 1995.

Caldwell, C. (1995) Telemedical trauma care. National Intormation Infrastructure Testbed Home Page.

Dakins, D. R. (1997) Utopian home healthcare vision lacks social reality. Telemedicine and TeleHealth Networks 3(1) 7-9. 
Emery, S. (1997) Evolving technology thwarts aim of cost analyses. Telemedicine and TeleHealth Networks 3(1) 20-25.

Flower, J. (1993) The other revolution in health care. Wired Ventures Ltd..

Global Telemedicine Report (1994) Global Telemedicine Report, July 1994, p. 14.

Hinsdale, K. (no date) Telemedicine trauma care. National Information Infrastructure Testbed Web Page.

Holaday, L. and Swett, H. (1995) Computing at Yale 9(3) December 1995

Jones, E. (1997) Industry observers predict growth trends spurred by Bellwether Bill. Telemedicine and TeleHealth Networks 3(1) 43-44.

Kim, Y, Cabral Jr., J.E., Parsons, D.M. et al (1995) SeaHawk: A Telemedicine Project in the Pacific Northwest. Department of Electrical Engineering, FT-10, University of Washington, Seattle, WA. 8/95 http://icsl.ee.washington.edu/projects/gsp9/spie95/seahawk.

Lenn, L. (1994) Telemedicine saves prisons money... Telemedicine Journal, April 1994.

Levy, D. (1994) Telemedicine extends reach of health care. USA Today, July 1994.

Masys, D. (1997) Telehealth: the need for evaluation. Journal of the American Medical Informatics Association 4(1) 69.

McGlinch, M. (1996) Telemedicine Applications at MAYO.

http://www.mayo.edu/telmed/app.html January 1996.

Michalecki, R. (1994) Connecting urban doctors and rural patients. Communications News 31, 2/1/94, pp26.

Moore, M. (1996) Telemedicine Demonstration Project, 1989-1992. Texas Tech MEDNET Telemedicine Project Summary, 2/19/1996.

O'Conner, M. (no date) Health Care Telecommunications Development at the University of Colorado Hospital and University of Colorado Health Sciences Center.

http://www.helmet,uhcolorado,edu

TARDIS Telemedicine Project (1998) (Telemedical Application for Remote Distributed Interactive Systems), http://www.dstc.edu.au/tardis/index.html

Unger, M. (1996) Digital DOCTORS/Physicians are practicing telemedicine... Newsday, January 1996. 